\title{
Yield and weed density of Blackgram (Vigna mungo (L.) Hepper) as influenced by weed control methods
}

\author{
S. Sahoo ${ }^{1 *}$, G.N. Dhanapal ${ }^{1}$, Pavankumar Goudar ${ }^{1}$, M.T. Sanjay ${ }^{1}$ and M.K. Lal ${ }^{2}$ \\ ${ }^{1}$ Department of Agronomy, College of Agriculture, University of Agricultural Sciences, Bangalore - 560065 \\ (Karnataka), INDIA \\ ${ }^{2}$ Division of plant physiology, IARI, New Delhi- 110012, INDIA \\ *Corresponding author. E-mail: mailsatyabrata.sahoo@gmail.com
}

Received: August 8, 2016; Revised received: December 12, 2016; Accepted: April 8, 2017

\begin{abstract}
The study conducted with pre-emergent herbicides alone and with sequential application of postemergent herbicides at All India Co-ordinated Research Project (AICRP) on weed management, Gandhi Krishi Vignyan Kendra (GKVK), Bangalore in late rabi season of 2013 revealed the predominance of grasses over broad leaved weeds in blackgram. Interculture @ 20 days after sowing (DAS) and hand weeding @ 40 DAS resulted in higher yield (1182 and $5873 \mathrm{~kg} \mathrm{ha}^{-1}$ seed and haulm yield, respectively) and least weed density of $41.33 \mathrm{~m}^{-2}$ during harvest. Uncontrolled weed growth recorded maximum population $\left(70.00 \mathrm{~m}^{-2}\right)$. During initial days pendimethalin 30

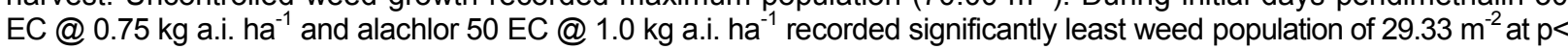
0.05 level of significance. Uncontrolled weed growth resulted in maximum reduction in yield of 65.64 per cent.
\end{abstract}

Keywords: Broad spectrum herbicide, Herbicide sequence, Yield reduction

\section{INTRODUCTION}

Blackgram is especially an unnoticed crop because of its cultivation as paira crop in rice fallows and as catch crop for catching the season where the main crop fails. Weeds, due to their competition with crop plants for nutrients, moisture, light and space cause yield reduction in pulses to greater extent. Unchecked weeds have been reported to cause a considerable reduction in the grain yield of black gram, which in case of summer and kharif blackgram could be 41.2 and 41.6 per cent, respectively (Singh, 2011). Therefore, removal of weeds at appropriate time using a suitable method is essential to obtain high yields of black gram.

Pre-emergent application of herbicide may allow the emergence of weeds, especially grasses after a few days. Due to which requirement of post-emergent herbicides for managing weeds is unavoidable. This investigation was planned and implemented to find out the influence of various weed control methods on yield as well as weed population of blackgram. For which the experiment was devised taking into account of the pre- as well as post-emergent herbicides so that weeds can be controlled throughout the crop growth period. Effect of various herbicides was compared with interculture followed by hand weeding and untreated check.

\section{MATERIALS AND METHODS}

The investigation was conducted at the field unit of Main Agricultural Research Station, Hebbal, during late rabi season of 2013. The region comes under the agro climatic zone southern plateau and hills with an elevation of $914 \mathrm{~m}$ Mean Sea Level. The soil textural class of the experimental site is sandy clay loam with low nitrogen, medium available phosphorus and available potassium status.

Type-9 cultivar of black gram was sown in line with a distance of $30 \mathrm{~cm}$ from row to row and $10 \mathrm{~cm}$ from plant to plant. The experiment comprised of 14 treatments which were replicated thrice. The treatments $T_{1}$ to $\mathrm{T}_{14}$ (Table 1) included pre-emergent herbicides alone and in combination with post-emergent herbicides along with interculture in combination with hand weeding and weedy check. Pre-emergent herbicides were sprayed 3 days following the date of sowing and similarly post-emergent herbicide spray was given 30 days following the date of sowing. Weed count was taken using a weed quadrant of $0.25 \mathrm{~m}^{2}$ at $15 \mathrm{DAS}, 45$ DAS and during harvest.

The yields from the net plots were taken in to account for yield estimation and were converted to hectare. The weed count values were subjected to square root transformation to make it normal distribution. The values obtained were subjected to Fisher's method of "Analysis of variance" (ANOVA) as outlined by Gomez and Gomez (1984) and data were compared with critical differences at a probability level of 0.05 per cent.

\section{RESULTS AND DISCUSSION}

Seed yield, haulm yield and weed index: Significant difference (P $0.05 \%$ ) was observed for seed as well as 
haulm yield (Table 2). Significantly higher seed and haulm yield was observed in interculture followed by hand weeding 1181.6 and $5873.3 \mathrm{~kg}^{-1}$, respectively and it was statistically on par with pendimethalin followed by quizalofop $\left(\mathrm{T}_{10}\right)$ with seed and haulm yield of 1120.6 and $5194.3 \mathrm{~kg} \mathrm{ha}^{-1}$, respectively. Seed and haulm yield was significantly reduced under unweeded check (405.4 kg of seed yield and $2700 \mathrm{~kg} \mathrm{ha}^{-1}$ ). The better performance of mechanical weeding was earlier reported by Brij et al. (2011) and Chhodavadia (2014) in greengram which might be due to nearly complete removal of weeds resulting in reduced crop weed interference thus crop growth was better which lead to more pod bearing branches and ultimately more haulm and seed yield. Among the herbicidal treatments the combination of pre- and post-emergent herbicides $\left(\mathrm{T}_{4}\right.$, $\mathrm{T}_{5}, \mathrm{~T}_{6}, \mathrm{~T}_{7}, \mathrm{~T}_{8}, \mathrm{~T}_{9}, \mathrm{~T}_{10}, \mathrm{~T}_{11}$ and $\mathrm{T}_{12}$ ) yielded more than single application of pre-emergent herbicides $\left(\mathrm{T}_{1}, \mathrm{~T}_{2}\right.$, $\mathrm{T}_{3}$ ). The reproductive sink depends on the vegetative source available due to which harvest index was moreover not influenced by weed control measures (Table $2)$. No control of weeds $\left(T_{14}\right)$ resulted in yield reduction of nearly 65.55 percent, similar findings were re- ported by Gogoi et al. (1991) where yield reduction ranged from 60 to 65 per cent in summer blackgram. Singh (2011) after conducting experiment for four years in blackgram reported that the yield reduction due to weed interference is 41.2 and 41.6 percent in summer and kharif season respectively.

Weed density: In the experimental plots population of grasses were maximum followed by broad leaved weeds with the least population being taken by sedges. Grasses and broad leaved weeds population differed significantly at 5 per cent level of significance throughout the crop growth period whereas, in sedges the influence of weed control practices were not effective.

At 15 DAS (Table 3) sedge and broad leaved weed population did not differ significantly. Pendimethalin 30 EC@ $0.75 \mathrm{~kg}$ a.i. ha ${ }^{-1}$ controlled grasses effectively and recorded least grass population of $6.67 \mathrm{~m}^{-2}$. Weedy check $\left(T_{14}\right)$ recorded significantly higher grass density of $31.33 \mathrm{~m}^{-2}$ at 5 per cent significance level. In summer season blackgram De and Modak (1993) also found that any weed control measures resulted in less population of weeds over the control. For total weed density

Table 1. Treatment details, dosage and time of application of herbicides.

\begin{tabular}{|c|c|c|}
\hline Treatments & Dosage $\left(\mathrm{kg} \mathrm{ha}^{-1}\right)$ & Time of application (DAS) \\
\hline $\mathrm{T}_{1}$-Pendimethalin $30 \mathrm{EC}$ & 0.75 & 3 \\
\hline $\mathrm{T}_{2}$-Alachlor $50 \mathrm{EC}$ & 1.0 & 3 \\
\hline $\mathrm{T}_{3}$-Oxyfluorfen $23.5 \mathrm{EC}$ & 0.075 & 3 \\
\hline $\mathrm{T}_{4}-\mathrm{T}_{1}$ fbimazethapyr $10 \mathrm{SL}$ & $0.75 \mathrm{fb} 0.063$ & $3 \mathrm{fb} 30$ \\
\hline $\mathrm{T}_{5}-\mathrm{T}_{2}$ fbimazethapyr $10 \mathrm{SL}$ & $1.0 \mathrm{fb} 0.063$ & $3 \mathrm{fb} 30$ \\
\hline $\mathrm{T}_{6}-\mathrm{T}_{3}$ fbimazethapyr $10 \mathrm{SL}$ & $0.075 \mathrm{fb} 0.063$ & $3 \mathrm{fb} 30$ \\
\hline $\mathrm{T}_{7}-\mathrm{T}_{1} \mathrm{fb}$ fenoxaprop-p-ethyl $9 \mathrm{EC}$ & $0.75 \mathrm{fb} 0.054$ & $3 \mathrm{fb} 30$ \\
\hline $\mathrm{T}_{8}-\mathrm{T}_{2} \mathrm{fb}$ fenoxaprop-p-ethyl $9 \mathrm{EC}$ & $1.0 \mathrm{fb} 0.054$ & $3 \mathrm{fb} 30$ \\
\hline $\mathrm{T}_{9}-\mathrm{T}_{3}$ fb fenoxaprop-p-ethyl $9 \mathrm{EC}$ & $0.075 \mathrm{fb} 0.054$ & $3 \mathrm{fb} 30$ \\
\hline $\mathrm{T}_{10}-\mathrm{T}_{1} \mathrm{fb}$ quizalofop-p-ethyl $5 \mathrm{EC}$ & $0.75 \mathrm{fb} 0.03$ & $3 \mathrm{fb} 30$ \\
\hline $\mathrm{T}_{11}-\mathrm{T}_{2} \mathrm{fb}$ quizalofop-p-ethyl $5 \mathrm{EC}$ & $1.0 \mathrm{fb} 0.03$ & $3 \mathrm{fb} 30$ \\
\hline $\mathrm{T}_{12}-\mathrm{T}_{3} \mathrm{fb}$ quizalofop-p-ethyl $5 \mathrm{EC}$ & $0.075 \mathrm{fb} 0.03$ & $3 \mathrm{fb} 30$ \\
\hline T13-Interculture@20 DAS and hand weeding@40DAS & - & - \\
\hline $\mathrm{T}_{14}$-Weedy check & - & - \\
\hline
\end{tabular}

$D A S$ - Days after sowing, fb-Followed by

Table 2. Seed yield, haulm yield, harvest index and weed index of blackgram as influenced by weed control practices.

\begin{tabular}{|c|c|c|c|c|}
\hline Treatments & $\begin{array}{l}\text { Seed yield } \\
\left(\mathrm{kg} \mathrm{ha}^{-1}\right)\end{array}$ & $\begin{array}{l}\text { Haulm yield } \\
\left(\mathrm{kg} \mathrm{ha}^{-1}\right)\end{array}$ & $\begin{array}{l}\text { Harvest } \\
\text { index }\end{array}$ & $\begin{array}{l}\text { Weed index } \\
(\%)\end{array}$ \\
\hline $\mathrm{T}_{1}$-Pendimethalin $30 \mathrm{EC} @ 0.75 \mathrm{~kg}$ a.i. ha ${ }^{-1},[\mathrm{PE}]$ & 719.3 & 4203.0 & 0.15 & 39.04 \\
\hline $\mathrm{T}_{2}$-Alachlor $50 \mathrm{EC} @ 1.0 \mathrm{~kg}$ a.i. ha ${ }^{-1},[\mathrm{PE}]$ & 759.3 & 4516.4 & 0.15 & 35.66 \\
\hline $\mathrm{T}_{3}$-Oxyfluorfen $23.5 \mathrm{EC} @ 0.075 \mathrm{~kg}$ a.i. ha ${ }^{-1},[\mathrm{PE}]$ & 734.0 & 4266.7 & 0.15 & 37.80 \\
\hline $\mathrm{T}_{4}-\mathrm{T}_{1}$ fbimazethapyr $10 \mathrm{SL} @ 0.063 \mathrm{~kg}$ a.i. ha ${ }^{-1},[\mathrm{POE}]$ & 767.9 & 4434.0 & 0.15 & 34.92 \\
\hline $\mathrm{T}_{5}-\mathrm{T}_{2}$ fbimazethapyr $10 \mathrm{SL} @ 0.063 \mathrm{~kg}$ a.i. ha ${ }^{-1},[\mathrm{POE}]$ & 868.8 & 4583.6 & 0.16 & 26.37 \\
\hline $\mathrm{T}_{6}-\mathrm{T}_{3}$ fbimazethapyr $10 \mathrm{SL} @ 0.063 \mathrm{~kg}$ a.i. ha ${ }^{-1},[\mathrm{POE}]$ & 1053.2 & 4787.5 & 0.18 & 10.75 \\
\hline $\mathrm{T}_{7}-\mathrm{T}_{1} \mathrm{fb}$ fenoxaprop-p-ethyl 9 EC @ 0.054 kg a.i. ha ${ }^{-1},[\mathrm{POE}]$ & 1078.2 & 4856.8 & 0.18 & 8.63 \\
\hline $\mathrm{T}_{8}-\mathrm{T}_{2} \mathrm{fb}$ fenoxaprop-p-ethyl 9 EC @ $0.054 \mathrm{~kg}$ a.i. ha ${ }^{-1},[\mathrm{POE}]$ & 1096.9 & 4719.3 & 0.19 & 7.04 \\
\hline $\mathrm{T}_{9}-\mathrm{T}_{3}$ fb fenoxaprop-p-ethyl 9 EC @ 0.054 kg a.i. ha ${ }^{-1}$, [POE] & 1078.6 & 4976.8 & 0.18 & 8.59 \\
\hline $\mathrm{T}_{10}-\mathrm{T}_{1} \mathrm{fb}$ quizalofop-p-ethyl 5 EC @ $0.03 \mathrm{~kg}$ a.i. ha ${ }^{-1},[\mathrm{POE}]$ & 1120.6 & 5194.3 & 0.18 & 5.03 \\
\hline $\mathrm{T}_{11}-\mathrm{T}_{2}$ fb quizalofop-p-ethyl 5 EC @ $0.03 \mathrm{~kg}$ a.i. ha ${ }^{-1},[\mathrm{POE}]$ & 853.9 & 4279.2 & 0.17 & 27.64 \\
\hline $\mathrm{T}_{12}-\mathrm{T}_{3} \mathrm{fb}$ quizalofop-p-ethyl $5 \mathrm{EC} @ 0.03 \mathrm{~kg}$ a.i. ha ${ }^{-1}$, [POE] & 985.9 & 4948.2 & 0.17 & 16.45 \\
\hline T $\mathrm{T}_{13}$-Interculture@20 DAS and hand weeding@40 DAS. & 1181.6 & 5873.3 & 0.17 & 0.00 \\
\hline $\mathrm{T}_{14}$-Weedy check. & 405.4 & 2700.0 & 0.13 & 65.64 \\
\hline S.Em \pm & 63.7 & 437.7 & 0.03 & NA \\
\hline $\mathrm{CD}(\mathrm{P} \leq 0.05)$ & 185.1 & 1377.2 & NS & NA \\
\hline
\end{tabular}


Table 3. Weed density (Number $\mathrm{m}^{-2}$ ) at 15 DAS in blackgram as influenced by weed control practices.

\begin{tabular}{|c|c|c|c|c|}
\hline Treatments & Sedge & Grasses & $\begin{array}{l}\text { Broad leaved } \\
\text { weeds }\end{array}$ & Total \\
\hline $\mathrm{T}_{1}$-Pendimethalin $30 \mathrm{EC} @ 0.75 \mathrm{~kg}$ a.i. ha ${ }^{-1},[\mathrm{PE}]$ & $2.18(4.67)$ & $3.37(10.67)$ & $3.86(14.00)$ & $5.50(29.33)$ \\
\hline $\mathrm{T}_{2}$-Alachlor 50 EC @1.0 kg a.i. ha ${ }^{-1},[\mathrm{PE}]$ & $1.77(3.33)$ & $3.15(10.00)$ & $5.01(24.67)$ & $6.20(38.00)$ \\
\hline $\mathrm{T}_{3}$-Oxyfluorfen $23.5 \mathrm{EC} @ 0.075 \mathrm{~kg}$ a.i. ha ${ }^{-1},[\mathrm{PE}]$ & $1.00(0.00)$ & $4.01(16.00)$ & $3.84(14.67)$ & $5.58(30.67)$ \\
\hline 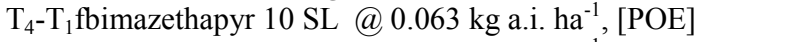 & $2.59(7.00)$ & $2.76(6.67)$ & $4.59(20.33)$ & $5.91(34.00)$ \\
\hline $\mathrm{T}_{5}-\mathrm{T}_{2} \mathrm{fbimazethapyr} 10 \mathrm{SL} @ 0.063 \mathrm{~kg}$ a.i. ha ${ }^{-1}$, [POE] & $1.00(0.00)$ & $3.60(12.00)$ & $4.27(17.33)$ & $5.51(29.33)$ \\
\hline $\mathrm{T}_{6}-\mathrm{T}_{3}$ fbimazethapyr $10 \mathrm{SL} @ 0.063 \mathrm{~kg}$ a.i. ha ${ }^{-1}$, [POE] & $1.67(2.67)$ & $4.82(22.67)$ & $4.80(22.00)$ & $6.93(47.33)$ \\
\hline $\mathrm{T}_{7}-\mathrm{T}_{1} \mathrm{fb}$ fenoxaprop-p-ethyl 9 EC @ $0.054 \mathrm{~kg}$ a.i. ha ${ }^{-1},[\mathrm{POE}]$ & $2.37(6.00)$ & $4.20(16.67)$ & $2.95(8.67)$ & $5.68(31.33)$ \\
\hline $\mathrm{T}_{8}-\mathrm{T}_{2} \mathrm{fb}$ fenoxaprop-p-ethyl 9 EC @ $0.054 \mathrm{~kg}$ a.i. ha ${ }^{-1},[\mathrm{POE}]$ & $1.96(3.33)$ & $3.83(14.00)$ & $3.73(13.33)$ & $5.60(30.67)$ \\
\hline $\mathrm{T}_{9}-\mathrm{T}_{3}$ fb fenoxaprop-p-ethyl 9 EC @ 0.054 kg a.i. ha ${ }^{-1}$, [POE] & $2.08(4.00)$ & $4.85(22.67)$ & $3.65(12.67)$ & $6.34(39.33)$ \\
\hline $\mathrm{T}_{10}-\mathrm{T}_{1} \mathrm{fb}$ quizalofop-p-ethyl 5 EC @ $0.03 \mathrm{~kg}$ a.i. ha ${ }^{-1}$, [POE] & $1.55(2.00)$ & $3.46(11.33)$ & $4.41(19.33)$ & $5.79(32.67)$ \\
\hline $\mathrm{T}_{11}-\mathrm{T}_{2} \mathrm{fb}$ quizalofop-p-ethyl 5 EC @ $0.03 \mathrm{~kg}$ a.i. ha ${ }^{-1},[\mathrm{POE}]$ & $1.41(1.33)$ & $3.91(14.67)$ & $3.93(16.00)$ & $5.73(32.00)$ \\
\hline $\mathrm{T}_{12}-\mathrm{T}_{3} \mathrm{fb}$ quizalofop-p-ethyl 5 EC @ $0.03 \mathrm{~kg}$ a.i. ha ${ }^{-1},[\mathrm{POE}]$ & $2.99(10.00)$ & $4.31(18.67)$ & $2.60(6.00)$ & $5.96(34.67)$ \\
\hline $\mathrm{T}_{13}$-Interculture@20 DAS and hand weeding@40DAS. & $1.41(1.33)$ & $5.54(30.00)$ & $3.77(13.33)$ & $6.75(44.67)$ \\
\hline $\mathrm{T}_{14}$-Weedy check. & $1.00(0.00)$ & $5.67(31.33)$ & $3.66(12.67)$ & $6.68(44.00)$ \\
\hline $\mathrm{S} . \mathrm{Em} \pm$ & 0.62 & 0.44 & 0.47 & 0.31 \\
\hline $\mathrm{CD}(\mathrm{P} \leq 0.05)$ & NS & 1.27 & NS & 0.91 \\
\hline
\end{tabular}

PE- Pre emergence at 3 DAS, POE- Post emergence at 30 DAS, fb- Followed by, DAS- Days after sowing, NS- Non significant, Figures in the parenthesis are original values; data analysed after square $\operatorname{root}(\mathrm{X}+1)$ transformation

Table 4. Weed density (Number $\mathrm{m}^{-2}$ ) at 45 DAS in blackgram as influenced by weed control practices.

\begin{tabular}{|c|c|c|c|c|}
\hline Treatments & Sedge & Grasses & $\begin{array}{ll}\text { Broad leaved } \\
\text { weeds }\end{array}$ & Total \\
\hline $\mathrm{T}_{1}$-Pendimethalin $30 \mathrm{EC} @ 0.75 \mathrm{~kg}$ a.i. ha ${ }^{-1},[\mathrm{PE}]$ & $1.96(3.33)$ & $4.57(22.67)$ & $4.27(18.00)$ & $6.55(44.00)$ \\
\hline $\mathrm{T}_{2}$-Alachlor 50 EC @1.0 kg a.i. ha ${ }^{-1},[\mathrm{PE}]$ & $1.96(4.67)$ & $4.25(17.33)$ & $5.55(30.00)$ & $7.27(52.00)$ \\
\hline $\mathrm{T}_{3}$-Oxyfluorfen 23.5 EC @ $0.075 \mathrm{~kg}$ a.i. ha ${ }^{-1},[\mathrm{PE}]$ & $1.55(2.00)$ & $5.50(30.00)$ & $4.97(24.67)$ & $7.58(56.67)$ \\
\hline 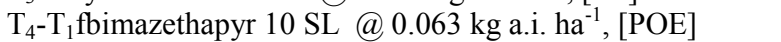 & $1.55(2.00)$ & $3.93(18.00)$ & $5.85(34.67)$ & $7.37(54.67)$ \\
\hline $\mathrm{T}_{5}-\mathrm{T}_{2}$ fbimazethapyr10 SL @ $0.063 \mathrm{~kg}$ a.i. ha ${ }^{-1}$, [POE] & $1.55(2.00)$ & $4.73(22.67)$ & $4.85(22.67)$ & $6.93(47.33)$ \\
\hline $\mathrm{T}_{6}-\mathrm{T}_{3}$ fbimazethapyr10 SL @ $0.063 \mathrm{~kg}$ a.i. ha ${ }^{-1}$, [POE] & $1.00(0.00)$ & $5.62(30.67)$ & $4.43(18.67)$ & $7.08(49.33)$ \\
\hline $\mathrm{T}_{7}-\mathrm{T}_{1} \mathrm{fb}$ fenoxaprop-p-ethyl 9 EC @ $0.054 \mathrm{~kg}$ a.i. ha ${ }^{-1},[\mathrm{POE}]$ & $2.37(6.00)$ & $4.25(20.00)$ & $5.88(35.33)$ & $7.88(61.33)$ \\
\hline $\mathrm{T}_{8}-\mathrm{T}_{2} \mathrm{fb}$ fenoxaprop-p-ethyl9 EC @ 0.054 kg a.i. ha ${ }^{-1}$, [POE] & $1.41(1.33)$ & $4.13(16.67)$ & $5.79(32.67)$ & $7.16(50.67)$ \\
\hline $\mathrm{T}_{9}-\mathrm{T}_{3} \mathrm{fb}$ fenoxaprop-p-ethyl 9 EC @ 0.054 kg a.i. ha ${ }^{-1}$, [POE] & $1.67(2.67)$ & $5.09(25.33)$ & $3.93(15.33)$ & $6.60(43.33)$ \\
\hline $\mathrm{T}_{10}-\mathrm{T}_{1} \mathrm{fb}$ quizalofop-p-ethyl 5 EC @ $0.03 \mathrm{~kg}$ a.i. ha ${ }^{-1},[\mathrm{POE}]$ & $1.55(2.00)$ & $3.13(11.33)$ & $5.91(34.00)$ & $6.90(47.33)$ \\
\hline $\mathrm{T}_{11}-\mathrm{T}_{2} \mathrm{fb}$ quizalofop-p-ethyl 5 EC @ $0.03 \mathrm{~kg}$ a.i. ha ${ }^{-1},[\mathrm{POE}]$ & $1.00(0.00)$ & $3.98(18.00)$ & $5.79(32.67)$ & $7.14(50.67)$ \\
\hline $\mathrm{T}_{12}-\mathrm{T}_{3} \mathrm{fb}$ quizalofop-p-ethyl 5 EC @ $0.03 \mathrm{~kg}$ a.i. ha ${ }^{-1},[\mathrm{POE}]$ & $1.67(2.67)$ & $4.63(22.00)$ & $4.53(21.33)$ & $6.78(46.00)$ \\
\hline $\mathrm{T}_{13}$-Interculture@20 DAS and hand weeding@40 DAS & $2.27(7.33)$ & $5.38(28.00)$ & $4.24(17.33)$ & $7.31(52.67)$ \\
\hline $\mathrm{T}_{14}$-Weedy check & $1.24(0.67)$ & $6.33(40.00)$ & $4.37(18.67)$ & $7.68(59.33)$ \\
\hline S.Em \pm & 0.66 & 0.87 & 0.65 & 0.65 \\
\hline $\mathrm{CD}(\mathrm{P} \leq 0.05)$ & NS & 2.52 & 1.61 & 1.63 \\
\hline
\end{tabular}

PE- Pre emergence at 3 DAS, POE- Post emergence at 30 DAS, fb- Followed by, DAS- Days after sowing, NS- Non significant, Figures in the parenthesis are original values; data analysed after square $\operatorname{root}(\mathrm{X}+1)$ transformation.

pendimethalin 30 EC@0.75 kg a.i. ha ${ }^{-1}$ and alachlor 50 EC@ $1.0 \mathrm{~kg}$ a.i. $\mathrm{ha}^{-1}$ recorded significantly least value of $29.33 \mathrm{~m}^{-2}$. While significantly higher total weed population was observed in oxyfluorfen $23.5 \mathrm{EC}$ (a) $0.075 \mathrm{~kg}$ a.i. ha ${ }^{-1}\left(47.33 \mathrm{~m}^{-2}\right)$ and was statistically on par with interculture@20 DAS and hand weeding@ 40 DAS $\left(44.67 \mathrm{~m}^{-2}\right)$ as well as $\left(\mathrm{T}_{14}\right)$ weedy check $\left(44.00 \mathrm{~m}^{-2}\right)$. Superior performance of pendimethalin due to its broad spectrum control was earlier reported by Jain and Jain (1987) in blackgram and Reddy et al. (2000) in rice-blackgram cropping sequence.

At 45 DAS (Table 4) Pendimethalin 30 EC @ 0.75 kg a.i. ha ${ }^{-1}$ followed by quizalofop-p-ethyl 5 EC @ 0.03 $\mathrm{kg}$ a.i. ha $\mathrm{ha}^{-1}\left(\mathrm{~T}_{10}\right)$ recorded least grass population of $11.33 \mathrm{~m}^{-2}$. During initial days pendimethalin controlled weeds and after 30 days where grasses start to emerge vigorously quizalofop being grass killer controlled grasses. The combination effect of pendimethalin and quizalofop were also reported by Kumar and Tewari (2004) in summer blakgram. Due to no control measures taken weedy check $\left(\mathrm{T}_{14}\right)$ recorded highest grass population of $40.00 \mathrm{~m}^{-2}$. Owing to physical removal of weeds twice, interculture@20 DAS and hand weeding@40 DAS ( $\left.\mathrm{T}_{13}\right)$ resulted in least population of broad leaved weeds $\left(17.33 \mathrm{~m}^{-2}\right)$. Pendimethalin 30 EC@0.75 kg a.i. ha ${ }^{-1}$ followed by fenoxaprop-p-ethyl9 EC @ $0.054 \mathrm{~kg}$ a.i. ha ${ }^{-1}\left(\mathrm{~T}_{7}\right)$ recorded highest broad leaved weed population of $35.33 \mathrm{~m}^{-2}$. During later part of crop growth dominance of grassy weeds are more and fenoxaprop being a grass killer controlled the grasses due to which broad leaved weeds were more which was not the case in weedy check where broad leaved weeds did not got a chance to grow freely due to dominance of grasses. When total 
Table 5. Weed density (Number $\mathrm{m}^{-2}$ ) at harvest in blackgram as influenced by weed control practices.

\begin{tabular}{|c|c|c|c|c|}
\hline Treatments & Sedge & Grasses & $\begin{array}{c}\text { Broad leaved } \\
\text { weeds }\end{array}$ & Total \\
\hline $\mathrm{T}_{1}$-Pendimethalin $30 \mathrm{EC} @ 0.75 \mathrm{~kg}$ a.i. ha ${ }^{-1},[\mathrm{PE}]$ & $1.00(0.00)$ & $1.29(18.00)$ & $1.48(28.67)$ & $6.90(46.67)$ \\
\hline $\mathrm{T}_{2}$-Alachlor 50 EC @ $1.0 \mathrm{~kg}$ a.i. ha ${ }^{-1},[\mathrm{PE}]$ & $1.00(0.00)$ & $1.46(28.00)$ & $1.39(22.67)$ & $7.18(50.67)$ \\
\hline $\mathrm{T}_{3}$-Oxyfluorfen $23.5 \mathrm{EC} @ 0.075 \mathrm{~kg}$ a.i. ha ${ }^{-1},[\mathrm{PE}]$ & $1.00(0.00)$ & $1.45(26.67)$ & $1.36(22.00)$ & $7.04(48.67)$ \\
\hline 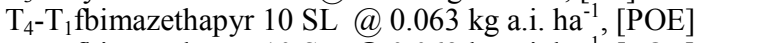 & $1.41(1.33)$ & $1.33(20.00)$ & $1.55(34.00)$ & $7.47(55.33)$ \\
\hline $\mathrm{T}_{5}-\mathrm{T}_{2}$ fbimazethapyr10 SL @ $0.063 \mathrm{~kg}$ a.i. ha ${ }^{-1},[\mathrm{POE}]$ & $1.00(0.00)$ & $1.53(32.00)$ & $1.31(18.67)$ & $7.19(50.67)$ \\
\hline $\mathrm{T}_{6}-\mathrm{T}_{3}$ fbimazethapyr10 SL @ $0.063 \mathrm{~kg}$ a.i. ha ${ }^{-1},[\mathrm{POE}]$ & $1.77(3.33)$ & $1.52(31.33)$ & $1.38(22.00)$ & $7.59(56.67)$ \\
\hline $\mathrm{T}_{7}-\mathrm{T}_{1} \mathrm{fb}$ fenoxaprop-p-ethyl 9 EC @ $0.054 \mathrm{~kg} \mathrm{a.i.h^{-1 }}{ }^{,}[\mathrm{POE}]$ & $1.00(0.00)$ & $1.27(16.67)$ & $1.53(32.67)$ & $7.09(49.33)$ \\
\hline $\mathrm{T}_{8}-\mathrm{T}_{2} \mathrm{fb}$ fenoxaprop-p-ethyl 9 EC $@ 0.054 \mathrm{~kg}$ a.i. ha $^{-1}$, [POE] & $1.00(0.00)$ & $1.28(17.33)$ & $1.49(28.67)$ & $6.85(46.00)$ \\
\hline $\mathrm{T}_{9}-\mathrm{T}_{3}$ fb fenoxaprop-p-ethyl 9 EC @ 0.054 kg a.i. ha ${ }^{-1}$, [POE] & $1.00(0.00)$ & $1.39(22.67)$ & $1.49(30.00)$ & $7.31(52.67)$ \\
\hline $\mathrm{T}_{10}-\mathrm{T}_{1} \mathrm{fb}$ quizalofop-p-ethyl $5 \mathrm{EC} @ 0.03 \mathrm{~kg}$ a.i. ha $^{-1},[\mathrm{POE}]$ & $1.00(0.00)$ & $0.93(10.67)$ & $1.55(33.33)$ & $6.70(44.00)$ \\
\hline $\mathrm{T}_{11}-\mathrm{T}_{2} \mathrm{fb}$ quizalofop-p-ethyl 5 EC @ $0.03 \mathrm{~kg}$ a.i. ha ${ }^{-1}$, [POE] & $1.00(0.00)$ & $1.47(28.00)$ & $1.37(21.33)$ & $7.08(49.33)$ \\
\hline $\mathrm{T}_{12}-\mathrm{T}_{3} \mathrm{fb}$ quizalofop-p-ethyl $5 \mathrm{EC} @ 0.03 \mathrm{~kg}$ a.i. $\mathrm{ha}^{-1}$, [POE] & $2.22(4.67)$ & $1.45(26.00)$ & $1.42(24.67)$ & $7.51(55.33)$ \\
\hline $\mathrm{T}_{13}$-Interculture@20 DAS and hand weeding @40 DAS & $1.00(0.00)$ & $1.39(22.67)$ & $1.31(18.67)$ & $6.49(41.33)$ \\
\hline $\mathrm{T}_{14}$-Weedy check & $1.00(0.00)$ & $1.74(55.33)$ & $1.21(14.67)$ & $8.39(70.00)$ \\
\hline $\mathrm{S} . \mathrm{Em} \pm$ & 0.29 & 0.1 & 0.06 & 0.29 \\
\hline $\mathrm{CD}(\mathrm{P} \leq 0.05)$ & NS & 0.29 & 0.16 & 0.85 \\
\hline
\end{tabular}

PE- Pre emergence at 3 DAS, POE- Post emergence at 30 DAS, fb- Followed by, DAS- Days after sowing, NS- Non significant, Figures in the parenthesis are original values; data analysed square root $(\mathrm{X}+1)$ transformation for sedge and total weed density, $\log (\mathrm{X}+2)$ transformation for grasses and broad leaved weed density.

weed population was considered oxyfluorfen $23.5 \mathrm{EC}$ (a) $0.075 \mathrm{~kg}$ a.i. $\mathrm{ha}^{-1}$ followed by fenoxaprop-p-ethyl 9 EC@ 0.054 kg a.i. ha ${ }^{-1}\left(\mathrm{~T}_{9}\right)$ recorded least weed density of $43.33 \mathrm{~m}^{-2}$. Oxyfluorfen is known for its residual effect due to which its effect was there for more duration than other herbicides and being a broad spectrum herbicide it controlled all three types of weeds and above that fenoxaprop being a grass killer controlled grasses more effectively which were dominant in the later part of crop growth. The better performance of grass killers along with a pre-emergent herbicides in blackgram were reported by Rao (2008), Tomar (2011), Mundra and Maliwal (2012) and Chhodavadia (2014) in summer green gram .While, pendimethalin 30 EC@0.75 kg a.i. ha ${ }^{-1}$ followed by fenoxaprop-pethyl 9 EC@0.054 kg a.i. ha ${ }^{-1}\left(\mathrm{~T}_{7}\right)$ recorded highest weed density of $61.33 \mathrm{~m}^{-2}$ which was solely due to more population of broad leaved weeds.

During harvest (Table 5), Pendimethalin 30 EC@ $0.75 \mathrm{~kg}$ a.i. ha ${ }^{-1}$ followed by quizalofop-p-ethyl $5 \mathrm{EC}$ (a) $0.030 \mathrm{~kg}$ a.i. ha ${ }^{-1}\left(\mathrm{~T}_{10}\right)$ recorded least grass density of $10.67 \mathrm{~m}^{-2}$ which was due to effective control of grasses during the earlier periods.Similar results were obtained by Kumar and Tewari (2004) where application of pendimethalin $\left(1.0 \mathrm{~kg}\right.$ a.i. $\left.\mathrm{ha}^{-1}\right)$ pre-emergence followed by fluazifop-p-butyl $\left(0.375 \mathrm{~kg}\right.$ a.i. $\left.\mathrm{ha}^{-1}\right)$ postemergence caused reduced weed growth as well as it resulted in 100 per cent mortality of Trianthema monogyna in summer blackgram. Whereas $\left(\mathrm{T}_{14}\right)$ weedy check resulted in highest grass population of $15.33 \mathrm{~m}^{-}$ ${ }^{2}$.Broad leaved weeds least density was obtained in $\left(\mathrm{T}_{14}\right)$ weedy check $\left(1.21 \mathrm{~m}^{-2}\right)$ where grasses smothered broad leaved weeds. While pendimethalin 30 EC @ $0.75 \mathrm{~kg}$ a.i. ha ${ }^{-1}$ followed by imazethapyr $10 \mathrm{SL} @$ $0.063 \mathrm{~kg}$ a.i. ha ${ }^{-1}\left(\mathrm{~T}_{4}\right)$ recorded highest weed density of $34.00 \mathrm{~m}^{-2}$. Interculture @20 DAS and hand weeding (a) 40 DAS $\left(\mathrm{T}_{13}\right)$ resulted in lower weed density of
$41.33 \mathrm{~m}^{-2}$ and the highest population of weeds were observed in $\left(\mathrm{T}_{14}\right)$ weedy check $70.00 \mathrm{~m}^{-2}$. Earlier works done by Kundu et al. (2011) and Chhodavadia (2014) in summer green gram are also in agreement with the superior performance of mechanical weeding in combination of manual weeding over other control measures.

\section{Conclusion}

It can be inferred that weeds cause 65.64 per cent yield loss if no weed control method is adopted. With abundant labour availability hand weeding at 20 DAS and intercultural at 40 DAS may be recommended for obtaining higher yield (1182 and $5873 \mathrm{~kg} \mathrm{ha}^{-1}$ seed and haulm yield, respectively) and reduced population of weeds $41.33 \mathrm{~m}^{-2}$. With the current trend of increased cost and reduced availability of manpower pendimethalin followed by quizalofop is the best option available for harvesting higher yield (seed and haulm yield of 1120.6 and $5194.3 \mathrm{~kg} \mathrm{ha}^{-1}$ ) as well as for controlling weeds $44.00 \mathrm{~m}^{-2}$.

\section{REFERENCES}

Singh, G. (2011). Weed Management in summer and kharif season blackgram [Vigna mungo (L.) Hepper]. Indian J. Weed Sci., 43(1\&2): 77-80

Gomez, K. A. and Gomez, A. A., (1984). Statistical procedures for agricultural research. John Wiley and sons, New Delhi, 680 p.

Brij, N., Kumar, A., Sharma, B.C. and Sharma, N. (2011).Chemical and cultural methods for weed control of mung bean under limited moisture conditions of Kandi belt of Jammu. Indian J. Weed Sci., 43(3\&4):241-242

Chhodavadia, S. K. (2014). Efficacy of pre- and post- emergence herbicides for integrated weed management in summer green gram (Vigna radiata L.).(Abstract) $2^{\text {nd }}$ Int. Conf. on Agric. and Hort. Sci., Hyderabad, India.

Gogoi, A. K., Kalita, H., Pathak, A. K. and Deka, J. (1991). Weed management in black gram. Indian J. Agron., 36 (4):601-602 
De, G. C. and Modak, R. (1993). Integrated weed management in summer blackgram (cv. B 76) in the lateritic tract of West Bengal. Proc. Indian Soc. of Weed Sci. Int. Symp., Hissar, India:150-151

Jain, N.K. and Jain, S.C. (1987). Impact of different weed free media on the growth and yield parameters of black gram (Vigna mungo) in semi-arid tropical conditions.Indian J. Weed Sci., 19(1\& 2): 20-24

Reddy, M.D., Reddy, C.N. and Devi, M.P. (2000).Effect of herbicides on weed growth and crop performance in rice -blackgram cropping system. Indian J. Weed Sci., 32 (3/4):169-172

Kumar, A. and Tewari, A. N. (2004). Crop-weed competition studies in summer sown blackgram (Vigna mungo L.). Indian J. Weed Sci.,36(1\&2):76-78
Rao, A. S. (2008).Effect of time and dose of post-emergence herbicides on Echinochloacolona (L.) in blackgram grown as relay crop. Indian J. Weed Sci., 40(3\&4):165168

Tomar, A. S. (2011).Evaluation of quizalofop-p-terfuryl $4.41 \%$ EC against grassy weeds in black gram (Vigna mungo L.). J. Crop and Weed, 7(1): 140-141

Mundra, S. L. and Maliwal, P. L. (2012) Influence of quizalofop-ethyl on narrow-leaved weeds in blackgram and its residual effect on succeeding crops. Indian $J$. Weed Sci., 44(4): 231-234

Kundu, R., Bera, P. S., Brhmachari, K. and Mallick, R. (2011). Integrated weed management in summer green gram (Vigna radiata L.) under gangetic alluvial soil of West Bengal. J. Botan. Soc. Bengal, 65(1):35-43 